\title{
Erratum to: Evolution of TOR and Translation Control
}

\author{
Bruno D. Fonseca, Tyson E. Graber, Huy-Dung Hoang, \\ Asier González, Alexander A. Soukas, Greco Hernández, \\ Tommy Alain, Stephanie L. Swift, Ronit Weisman, Christian Meyer, \\ Christophe Robaglia, Joseph Avruch and Michael N. Hall
}

\section{Erratum to:}

"Evolution of TOR and Translation Control" in: G. Hernández and R. Jagus (eds.), Evolution of the Protein Synthesis Machinery and Its Regulation, DOI 10.1007/978-3-319-39468-8_15

The subjected book was published with the following errors:

- An incorrect spelling of the author's name as "Alexandre A. Soukas" in Chap. 15 and in the table of contents. It should be "Alexander A. Soukas".

The updated original online version for this chapter can be found at 10.1007/978-3-319-394688_15

B.D. Fonseca $(\bowtie) \cdot$ T.E. Graber · H.-D. Hoang · T. Alain · S.L. Swift

Children's Hospital of Eastern Ontario Research Institute, 401 Smyth Road, Ottawa, ON K1H 8L1, Canada

e-mail: brunodfonseca@gmail.com

A. González · M.N. Hall

Biozentrum, University of Basel, 4056 Basel, Switzerland

A.A. Soukas · J. Avruch

Massachusetts General Hospital, Boston, MA, USA

G. Hernández

Division of Basic Science, National Institute of Cancer, 22 San Fernando Avenue, Tlalpan, 14080 Mexico City, Mexico

R. Weisman

Department of Molecular Microbiology and Biotechnology, Tel Aviv University, Tel Aviv, Israel

R. Weisman

Department of Natural and Life Sciences, The Open University of Israel, Raanana, Israel 
- A figure was placed incorrectly on p. 354 whereas it should have been placed on p. 366.

- A typographical error occurred on page 358 (line no 988). The spelling of a word was given as "nicotic" whereas it should be "nicotinic".

The erratum has been updated in the book and chapters.

C. Meyer

Institut Jean-Pierre Bourgin, Unité Mixte de Recherche 1318, Institut National de la

Recherche Agronomique, AgroParisTech, ERL CNRS 3559, Saclay Plant Sciences, 78026

Versailles Cedex, France

C. Robaglia

Aix-Marseille Université, Laboratoire de Génétique et Biophysique des Plantes, 13009

Marseille, France

C. Robaglia

CNRS, UMR 7265 Biologie Végétale \& Microbiologie Environnementales, 13009 Marseille,

France

C. Robaglia

CEA, IBEB, 13009 Marseille, France 